\title{
An Olfactory-Based Robot Swarm Navigation Method
}

\author{
Ali Marjovi, João Nunes, Pedro Sousa, Ricardo Faria, Lino Marques
}

\begin{abstract}
This paper presents a novel robot swarming navigation algorithm in order to find the odor sources in an unknown environment, based on the ability of each swarm member to sense the odor. Each robot in the swarm has a cooperative localization system which uses wireless network as a mean of measuring the distance from the other robots. In this method, at least three robots act as stationary measurement beacons while the other robots of the swarm navigate in the environment towards the odor source. In the next step, the roles of the robots will be switched and some other robots will act as beacons. The experimental tests report a good result in finding the odor source and also the accuracy of localization system ${ }^{1}$.
\end{abstract}

\section{INTRODUCTION}

Olfaction plays a significant role in the way of life of most animals, being particularly important for finding food, avoiding threats and coordinating behaviors in social animals. Although playing a major role in natural life, olfaction has been a disregarded sense inside the robotics community since only few groups have researched about its integration relatively in mobile robots [1].

The possibility to measure the gas concentration with a mobile robot enables a broad range of applications, ranging from surveillance of environmental pollutants and the detection of hazardous gases, to self-produced odors for aiding navigation [2], [3]. Odor localization is regarded as the base of many applications using olfactory mechanisms [4]. So far, the main problem studied in olfactory navigation has been the tracking and localization of static odor sources [4], [5]. In real situations, this problem can be broken into three sub-problems: finding traces of the chemical of interest; tracking the respective odor plume; and localizing the odor source. Odor plume tracking has been the problem more deeply studied using heuristic and bio-inspired algorithms, odor tracking sometimes complemented by other sensing modalities, like vision [5].

The mobile robot cooperation using olfaction is a less studied field. Some reference works in this area are: the collaborative spiral surge algorithm proposed by Hayes and co-workers for finding odor sources with a group of robots [6]; the cooperative area coverage using olfaction that was addressed by [7] proposing an online complete coverage algorithm based on the utilization of chemical markings and a biologically-inspired algorithm for gas/odor source localization in an indoor environment with no strong airflow

\footnotetext{
Institute of Systems and Robotics, University of Coimbra, Portugal\{ali, jgnunes, pvsousa, rfaria, lino\}eisr.uc.pt

${ }^{1}$ This work was partially supported by project GUARDIANS contract FP6-IST-045269 and also by Portuguese Foundation for Science and Technology contract SFRH/BD/45740/2008.
}

by [8]. The problem of finding traces of odor plumes in large search spaces has been addressed in [9] using a group of mobile robots coordinated by a particle swarm-based algorithm (PSO). [10] introduced a small group of robots and a Kalman-based data assimilation techniques to estimate the distribution of a transient odor field inside a laboratory. A major drawback of their method was its centralized approach.

Swarm research and swarm robotics is a relatively new field that has gained momentum since the pioneering work by Reynolds [11] on simulation of a flock of birds in flight. However olfaction has not been a well-developed field inside the swarming robotics community since only relatively few groups have researched its integration in mobile robots.

In the other hand, localization approach is a significant issue for the navigational aspect of most robotic applications. To the best of the authors' knowledge, none of the mentioned researchers above have addressed the problem of localization in olfactory-based swarming. Without accurate positioning, a mobile robot would wander away from its target workspace and would fail to complete its planned task. There are many situations where an external positioning system, such as GPS, is unavailable to the robots. A team of robots can employ cooperative localization to incorporate relative sensor measurements into a Kalman filter framework that estimates the pose of the robots [19]. Many of the cooperative localization systems, using infrared, ultrasound and $\mathrm{Wi}-\mathrm{Fi}$, have been developed for indoor localization after GPS but each of them shows its merits and weaknesses at the same time. Infrared is applicable only to open spaces because of its characteristics of going straight. If the signal meets the obstacle, it easily reflects itself, which causes a difficulty in interpreting signal data. Using ultra-sound, it is easy to estimate the distance in proportion to the velocity of $330 \mathrm{~m} / \mathrm{s}$. However it takes long time to compute comparing to electronic signals' high speed. Wi-Fi ,known as wireless LAN, is hardly applicable to frequent mobile devices. ZigBee is very cheap and less power consuming wireless techniques comparing to other types such as RFID, infrared and ultrasound [20].

Tully et. al. [19] presented a "leap-frog" method for a team of three robots performing cooperative localization during navigation. In that method two robots act as stationary measurement beacons while the third moves in a path that provides informative measurements. After completing the move, the roles of each robot are switched and the path is repeated. They demonstrated accurate localization using this method in a coverage experiment in which three robots successfully swept a $20 \mathrm{~m} \times 30 \mathrm{~m}$ area. They claimed that it is one of the largest successful GPS-denied coverage experiments to that date. 


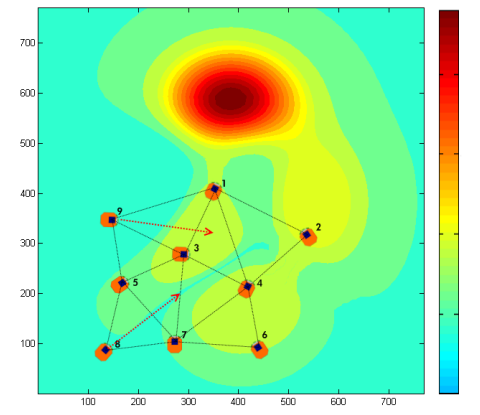

Fig. 1. A swarm of robots navigating toward the odor source while measuring distance from each other in order to maintain cooperative localization system.

An important part in this research is the measurement of odor concentration by the robots. Authors have addressed this issue in previous papers [21], [22] and [9].

In this research, an approach for efficient swarm navigation algorithm using olfactory-based steering for terrain surveying by spatial concentration of odor fields is presented and the ability to estimate the location of odor source in continuous odor fields from sparse data taken with a group of mobile sensing robots is addressed. Using a cooperative approach for positioning system, this method addresses the problem of localization of the robots. In the flowing sections, the suggested method for "localization and communication", "odor concentration measurement" approach and "decision making" algorithm are demonstrated. Finally, experimental results and conclusions are discussed.

\section{Problem Statement}

Consider a system of $\mathrm{N}$ mobile robots, moving in $R^{2}$ that are labeled as $A_{1}, A_{2}, \ldots, A_{N}$. Each agent $A_{i}(i \in\{1, \ldots, N\})$ is able to communicate with the other robots in a short distance range. An odor source is in the environment which is emitting odor gas into the area. All the robots are equipped with odor sensors for sensing the odor concentration. Robots are limited in terms of memory capacity and there is no central station for the system. The robots should act separately and independently from the others. There are no global positioning systems, the odometry of the robots is not reliable and robots do not have any accurate internal system for localization. The problem is how the swarm of robots can localize the odor source in the area (Fig. 1).

The approach should exploit particle swarm optimization with multiple robots to find odor source in natural environment where the odor distribution may change over time.

\section{PROPOSED METHOD}

In the proposed plan, each robot determines its pose based on a cooperative method that is described in the following section and it also measures the concentration of odor in that location using its sensor board that is described in this paper. Having all this data, each robot in the swarm runs a behavioral distributed algorithm and makes a decision. The decision making algorithm is described in the last part of this section.

\section{A. Localization}

Fusing the leap-frog method [19] and the signal strength of a ZigBee network [20] as the measuring tool and also as communication media for the robots in the swarm; a cooperative localization and communication system is developed.

In this method, during each period of time several robots separately take actions and move for a short distance while at least three other robots act as stationary measurement beacons. In some conditions, the roles of robots are switched and probably three other robots will act as beacons and the rest of the swarm moves to the direction of estimated target.

Each robot is equipped with a ZigBee module for communication network, it is also used for measuring the distance of the robot with the other robots. The communication modules were configured to operate in broadcast mode and their power levels were reduced to the minimum. These adjustments allow us to exchange information directly between robots and also to obtain a higher ratio between RF power loss and distance. By using the Received Signal Strength Indicator (RSSI) it is possible to estimate the distance between a transmitter and a receiver. We used a classic model of propagation loss based on the signal's travelling distance. The model is defined as [23];

$$
P(d)=P o\left(d_{0}\right)+10 n \log \left(\frac{d}{d_{0}}\right)+N(0, \sigma)
$$

where $P(d)$ is the "received power at distance d", $P o\left(d_{0}\right)$ is the "reference received power at distance $d_{0}$ ", $n$ represents the "path loss exponent" and $N(0, \sigma)$ is "normal distribution with zero mean and $\sigma$ standard deviation".

In this research we used the same model, obtained from linear regression, but instead of distance we used the dutycycle of a PWM signal generated by the radio module. The XBee modules generate a $15.6 \mathrm{kHz}$ PWM signal with a duty-cycle proportional to the received power.

The model that relates the duty-cycle with the distance was obtained based on empirical results. The measurements were obtained with two XBee modules (one emitter and one receiver), exchanging a 64 byte data packet with $30 \mathrm{~cm}$ increment between measurements, the measured raw data is shown in Fig. 2. Analyzing the data shown in Fig. 2 and using the Least Squares Fitting method to determine a second order polynomial equation for representing the relation between "distance of sender and receiver" and "measured PWM duty cycle",

$$
T_{\text {on }}(d)=57.3116-0.5876 d-0.3842 d^{2}+N(0, \sigma)
$$

where $T_{o n}$ is "pulse duration (time of high signal) in each period of received signal" and $d$ is "distance in meters".

Since the distance obtained from RSSI measurements is affected by unknown errors, in order to have a better position estimation, a particle providing position estimation based on robots odometry and RSSI measurements was used.

J. Rodas et. al. [23] introduced an algorithm based on particle filters for localization. They have used RSSI of 


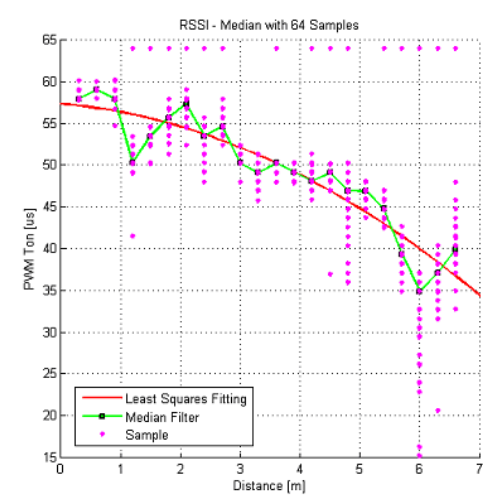

Fig. 2. "Pulse duration" of the received signal in different distances

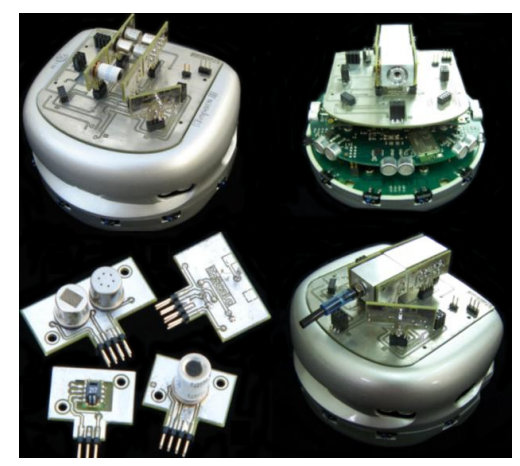

Fig. 4. Khepera III and KheNose with sensing modules

bluetooth communication to estimate the distance between transmitter and receiver. In this project we used the same method with some modifications for adapting it to ZigBee, based on multiple robots and using equation (2) as our propagation model.

Localization algorithm: As previously stated, the localization system uses RSSI to estimate the distance from the source. It is possible to use static robots acting as stationary beacons while the moving robots are the receiver. Using a very simple geometric calculation each robot is able to find out its location based on the position of the beacons (Fig. 3).

\section{B. Odor concentration measurement}

KheNose is an olfactory system composed of six transducer interface modules: an eCO, three thermal anemometers, and two eNostrils [24]. The eCO and the anemometers are single channel IEEE1451.4 compliant boards and the eNostrils are double channel boards. All the functions related with the transducers, namely signal conditioning, data acquisition and processing and calibration management are performed by the kheNose board. The Khenose was designed and implemented by the authors specially to be used in Khepera III robots, however for this project we used it even in the other robots that were involved in the experiments (Fig. 4).

\section{Decision making method}

A swarm of robots are spread in the environment, each one has a sensor and is able to send and receive data to others; this means that a mobile sensor network is established. By analyzing the concentration of odor in the environment, the odor source location can be estimated. However, there is no central unit to decide where the swarm should go, therefore each robot has to analyze the date itself and take an action. The action that each particle in the swarm takes will affect the behavior of all the other particles and it must be somehow in order to taking a step toward solving the problem (finding the odor source). It means that the decision making is distributed among the robots and thus each robot should independently decide and take an action in order to approach the odor source.

Each robot has a status record including its position and also the olfactory environmental data acquired from its sensors. Due to localization method, using ZigBee wireless network, each agent is able to request for the status record of the other agents. Since our localization method is a kind of leap-frog approach, at least three of the robots must act as stationary beacons every time. After a short time the role of the robots should be switched and three other robots must act as beacons. The lack of a central station makes it difficult in terms of implementation. Fig. 5 shows the state flow diagram of a robot's behavior in the swarm. There are four states that a robot can take in this method; "Scatter", "Aggregate", "Beacon" and "Odor source localization". All the robots start initially in the "Beacon" state, they change their status to the other states according to the conditions described below.

1) "Scatter": In this phase, the robots spread and scatter in the environment while trying to maintain cohesion. Each robot actually tries to get far from the neighbors but always takes into account being in the range of the swarm's beacons.

This simply is implemented by a potential field algorithm where each robot considers the other robots as repulsive forces and tries to minimize the summation of these repulsive vectors by moving in the environment.

In this state, robots communicate with each other by sending and receiving their pose and olfactory data. If three of the robots proclaim a high degree of concentration of the odor in their location (greater than a threshold), the agents change their state to "Beacon" state (transition (2) showed in the diagram, Fig. 5), making it possible to go to the "Aggregate" state in the next decision.

2) “Beacon": A Robot in this state acts as a stationary beacon providing localization system by broadcasting ping messages to the other robots. All the robots are allowed to move except only three of them (beacons); the ones which have the top three highest concentration of the odor in the environment. Therefore, every robot should receive and analyze the data of the other robots and see if its value is one of the top three values. In this case, the robot must stop and act as a beacon (transitions (2), (4) and (6) in the diagram); otherwise it is free to move, either in "Scatter", "Aggregate" or "Odor source localization" states (algorithm 1, lines 22 to 32). 


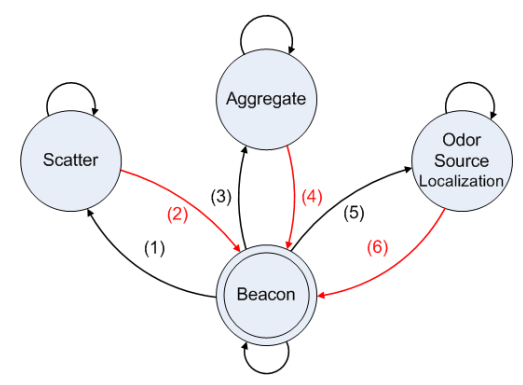

Fig. 5. State diagram of proposed algorithm

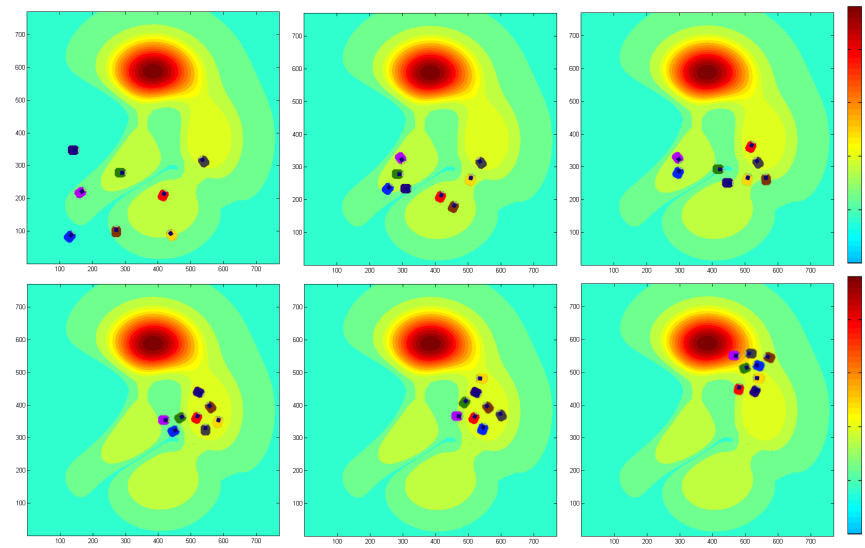

Fig. 6. 6 snapshots of simulation screen; every time 3 robots are stop and the others are moving on.

While the robot is moving, it should always take into account the distance with the beacons. If the robot is getting far from the stationary beacons, it has to stop and give the turn to another robot to move. This method assures that always the robots which are in the lower concentration of odor will try to improve their behavior in the swarm (see the simulation screen shots shown in Fig. 6). If all the robots announce a very low odor concentration (it means that they have lost the plume track) all of them go to "Scatter" state (transitions (1)), otherwise the robot goes to "aggregate" state when it is not one of the top three robots in terms of odor concentration anymore (transition (3)).

3) "Aggregate": In this state, it is desired for the robot to navigate to the direction of the odor source. Having the measured data of different places of an environment, extrapolation algorithms (like Kriging) can approximate where the odor source is. But the agents are limited in memory capacity and processing capability, therefore they can not run heavy algorithms for analyzing the data. In the other hand, if each agent in the swarm takes a small step towards the source, all the swarm will coverage to the source location.

In this method, each robot randomly chooses one of the three robots which have highest concentration of the odor in the swarm and considers a virtual vector to that direction as its orientation for this movement.

The robot has to stop (change to "Beacon" state) in three cases; first if the robot is getting far from at least three stationary beacons so that the signal strength reaches to a specific low threshold, second if the concentration of odor in the surrounding area is less than a specific threshold and the third if its odor concentration has reached to a point that this robot is one of the top three in the swarm. In these three instances the robot stops to be a temporary beacon for the others (algorithm 1, lines 17 to 21). In some conditions it will have the chance to make a decision and move on (transitions (1), (3) and (5)).

4) "Odor source localization": A robot has been facing to a very high odor concentration before coming to this state. In this state, each robot separately takes several short movements and estimates the position of the odor source by a kind of spiral movements. A robot in the plume moves straight upwind until it looses the plume for a specific distance. It then should try to reacquire the plume by moving along an Archimedean spiral with a specific gap size until it reacquires the plume [25].

The authors have addressed the problem of localizing multiple odour sources scattered across a search area by proposing an online searching method based on evolutionary techniques [3]. For finding another odor source, the robots go back to the "Beacon" state (transition (6) in the state flow diagram Fig. 5) making it possible to go to the "Scatter" or "Aggregate" state in the next decision.

Flocking: Algorithm 1 presents the proposed behavioral based algorithm for a single robot in the swarm. In the lower layer of this system, each robot implements obstacle avoidance during its navigation. The robots should maintain a specific distance from each other, therefore they must avoid crowding neighbors, this is called "separation behavior" in flocking modeling. "Alignment" and "cohesion" are the other two behaviors for a robot in a flock. Potential field technique is used to ensure these behaviors in each robot. Our potential field algorithm also guarantees "separation" (short range repulsion) and "cohesion" (long range attraction) behaviors.

\section{EXPERIMENTS}

The method has been simulated and then tested in the real world. For optimizing the exploration algorithm and measuring its performance, we used the Player/Stage simulator [26]. In the real world, there are a lot of constraints that do not let us test the proposed method very easily. It is not effortless to work with a lot of different robots with different scales, in the other hand we currently do not have enough hardware resources to test the method in a large scale in the real world. For these reasons, the system is developed and evaluated in the simulation before the real world experiments.

Two Khepera III $^{1}$ robots and two Erratic Robots ${ }^{2}$ were used for testing the algorithm. The robots were equipped with several infra-red and sonar sensors. These sensors are used for obstacle avoidance and navigation in potential field subsystem. Robots are equipped by ZigBee modules providing communication and also localization. The radio

\footnotetext{
${ }^{1}$ produced by K-Team SA, Switzerland

${ }^{2}$ produced by Videre Design LLC, California, USA
} 


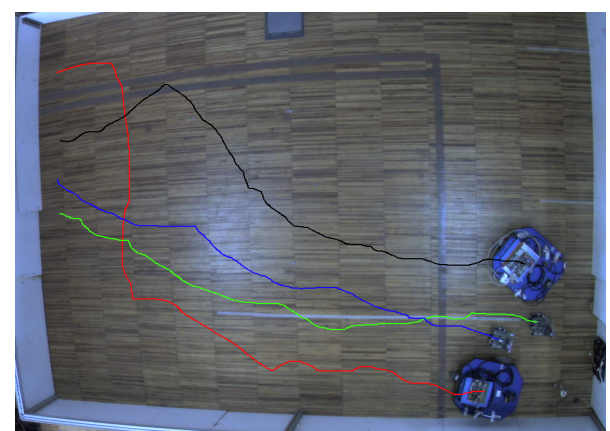

Fig. 7. The real world experiments, 2 Erratic and 2 Khepera robots localizing the odor source that in the bottom-right corner of the picture. The colored lines show the footprint of the robots.

modules used for localization were the Maxstream XBee 802.15.4 RF. These modules use the IEEE 802.15.4 networking protocol and provide wireless end-point connectivity with high-throughput and low latency in a range of up to $30 \mathrm{~m}$ indoor and $90 \mathrm{~m}$ outdoor in line of sight. All the robots are equipped with KheNose boards for gas source detection.

Variant testing plans were tested, having different start positions for the robots. One of the experimental setups is depicted in Fig. 7. It has an area of $3 m \times 4 m$ and there exist a pipe connected to a gas source that is releasing ethanol in the environment. There is a fan in the corner that generates air flow in the system.

Fig. 7 shows four robots exploring area and finding an odor source. The footprints show the paths taken by the robots. As it is shown, the robots have converged to the odor source. The localization algorithm does not allow all the robots moving at the same time, since they are only four, only one of them moves at a time and the others act as beacons.

Air flow and sensing uncertainty are the two main causes to have different results in similar tests. A parameter for evaluation of the method is the total mission time. The mission time for the environment shown in Fig. 7, using four robots, was 922 seconds. The result is the average of five similar tests. Different tests with constant conditions had similar results with about 8 variance. In all the tests the maximum speed of the robots were kept constant. The difference between the internal position value of each robot (coming from the cooperative localization system) and the real location of the robot (measured manually) after the mission was less than 10 centimeters. These results proves the functionality of the whole proposed method.

In order to have a better knowledge about the performance of this method, Player/Stage was used as the simulator. Since there is no tool for simulating an odor source in the Player/Stage, we measured the odor concentration in a real testing environment by setting up a sensor network and acquiring its data. This data was saved in a file as a dataset of odor distribution of a real environment. This file was used in Player/Stage as the distribution of the odor in the simulation world. Therefore, there is no air flow in the simulation but at least the method can bed tested in a pseudo-realistic way.

In simulation, the environment shown in Fig. 6 has been
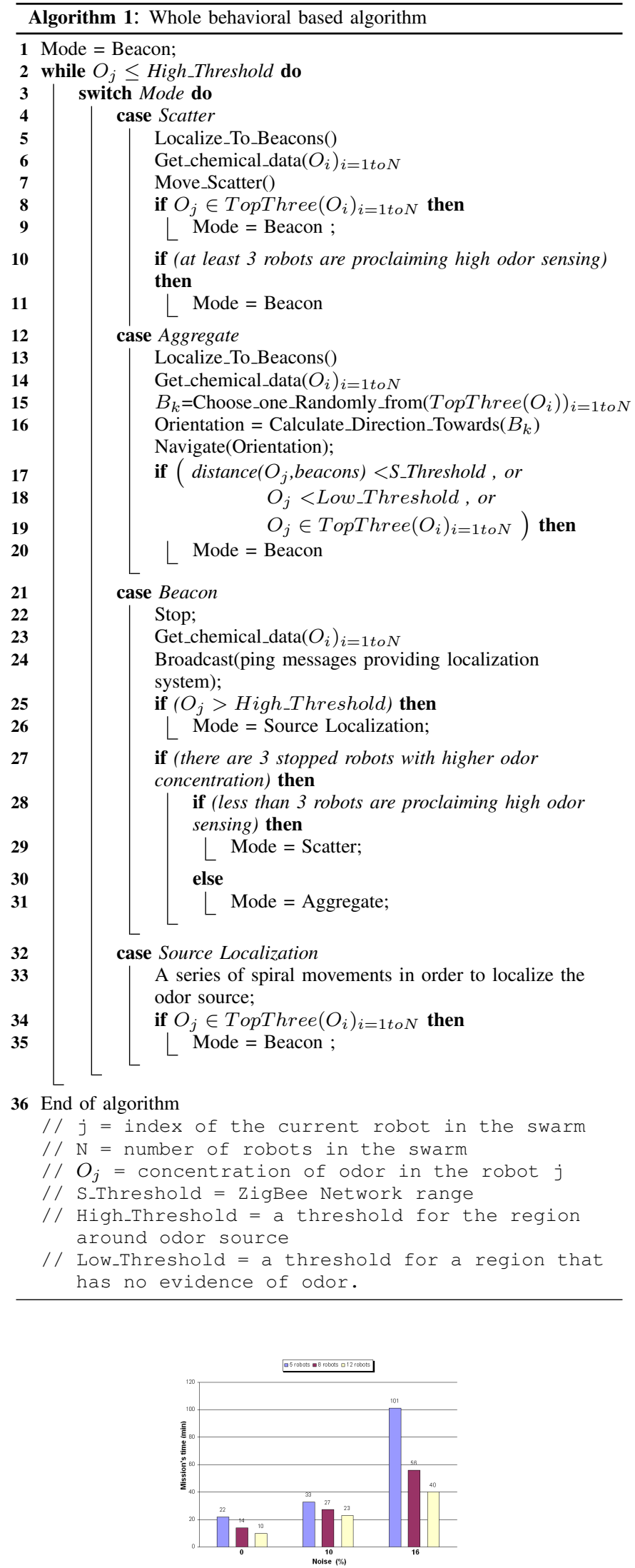

Fig. 8. The results of simulation in Player/Stage using different number of robots with variant noise distortion. Area dimension $=30 \mathrm{~m} \times 24 \mathrm{~m}$, maximum speed of the robots $=0.2 \mathrm{~m} / \mathrm{s}$ 
tested with 5, 8 and 12 robots. A good way for proving the functionality and fault tolerance of the method is adding noise to the odor data and testing the system. The algorithm was tested with adding 0,10 and 16 percent noise (based on experimental data) to the odor data and with different numbers of robots in the swarm. The results are shown in Fig. 8. The graph shows the average of five times tests for each dataset. The variance was about 6,9 and 10 percent (for 0,10 and 16 percent odor noise in the environment). This chart shows that a bigger swarm has a better result even in a noisy environment.

\section{CONCLUSIONS AND FUTURE WORKS}

This paper presented an olfactory based navigation algorithm for a swarm of robots in order to find an odor source in the environment. This algorithm is designed based on the ability of smelling, communicating with other robots and avoiding the obstacles for each robot. In this method, at least three of the robots of the swarm act as temporary beacons to guarantee the accuracy of positioning system of all the robots. The beacons exchange their roles with mobile robots in certain defined conditions in the algorithm. The cooperative localization system in each robot uses RSSI of ZigBee messages to measure the distance of the robot from the beacons and then profits from a particle filter to estimate its real current position. The algorithm was first simulated in the Player/Stage with a group of 5,8 and 12 robots. Then it was physical tested in a testing setup with four real robots. Reliability of the method against noise in the odor measuring (sensory uncertainty) has been evaluated and the results showed that the method is fully functional with a good performance.

We intend to test the method with more robots in multiple real environments. The particles in the swarm do not use any past record in their processing (because it was supposed to be a memory less state flow algorithm), but taking some past data into account for each robot (especially in the aggregation mode) will increase the performance of the method.

\section{REFERENCES}

[1] P. Lopalco, S. Lobasso, A. Corcelli, M. Dibattista, R. Araneda, P. Z., and S. Firestein, "Do olfactory receptors respond to explosives?" in IEEE Int. Workshop on Advances in Sensors and Interface, Italy, 2007.

[2] L. Marques and A. T. de Almeida, "Editorial to mobile robot olfaction," Autonomous Robots Journal, Special Issue on Mobile Robot Olfaction, Kluwer, vol. 20, no. 3, pp. 183-184, 2006.

[3] L. Marques, U. Nunes, and A. T. de Almeida, "Odour searching with autonomous mobile robots: An evolutionary-based approach," in IEEE Int. Conf. on Advanced Robotics, Taiwan, 2003.

[4] C. Xin and L. Yangmin, "Odor localization using swarm intelligence," in IEEE Int. Conf. on Robotics and Biomimetics, Hong Kong, 2005.

[5] L. Marques, U. Nunes, and A. T. de Almeida, "Olfaction-based mobile robot navigation," Thin Solid Films, Elsevier Science, vol. 418, no. 1, pp. 51-58, 2002.

[6] A. T. Hayes, A. Martinoli, and R. M. Goodman, "Distributed odor source localization," IEEE Sensors Journal, Special Issue on Artificial Olfaction, vol. 2, no. 3, pp. 260-271, 2002.

[7] S. Larionova, N. Almeida, L. Marques, and A. T. de Almeida, "Olfactory coordinated mobile robot area coverage," Autonomous Robots Journal, Special Issue on Mobile Robot Olfaction, Kluwer, vol. 20, no. 3, pp. 251-260, 2006.
[8] G. Ferri, E. Caselli, V. Mattoli, A. Mondini, B. Mazzolai, and P. Dario, "Spiral: A novel biologically-inspired algorithm for gas/odor source localization in an indoor environment with no strong airflow," Robotics and Autonomous Systems Journal, vol. 57, no. 4, 2009.

[9] L. Marques, U. Nunes, and A. T. de Almeida, "Particle swarm-based olfactory guided search," Autonomous Robots Journal, Special Issue on Mobile Robot Olfaction, Kluwer, vol. 20, no. 3, pp. 277-287, 2006.

[10] L. Marques, A. Martins, and A. de Almeida, "Environmental monitoring with mobile robots," in IEEE/RSJ Int. Conf. on Intelligent Robots and Systems (IROS), Canada, 2005.

[11] C. W. Reynolds, "Flocks, herds, and schools: A distributed behavioral model," in 14th Annual Conf. on Computer Graphics and Interactive Techniques, vol. 21, no. 4, New York, USA, 1987.

[12] D. P. Stormont and A. Kutiyanawala, "Localization using triangulation in swarms of autonomous rescue robots," in IEEE Int. Workshop on Safety, Security and Rescue Robotics, Italy, 2007.

[13] E. Bahceci and E. Sahin, "Evolving aggregation behaviors for swarm robotic systems: A systematic case study," in IEEE Swarm Intelligence Symposium, California, USA, 2005.

[14] V. Gazi and K. M. Passino, "Stability analysis of social foraging swarms," in IEEE Trans. on Systems, Man, and Cybernetics, vol. 34, no. $1,2004$.

[15] R. Bachmayer and N. E. Leonard, "Vehicle networks for gradient descent in a sampled environment," in IEEE Conf. on Decision and Control, Nevada, USA, 2002.

[16] O. Soysal and E. Sahin, "Probabilistic aggregation strategies in swarm robotic systems," in IEEE Swarm Intelligence Symposium, California, USA, 2005.

[17] S. Patnaik, A. Konar, and A. K. Mandal, "Improving the multi-agent coordination through learning," IETE Journal of Research, vol. 51, no. 5, pp. 395-406, 2005.

[18] V. Kumar, D. Rus, and S. Singh, "Robot and sensor networks for first responders," IEEE Pervasive computing Journal, pp. 24-33, 2004.

[19] S. Tully, G. Kantor, and H. Choset, "Leap-frog path design for multirobot cooperative localization," in Int. Conf. on Field and Service Robots, Massachusetts, USA, 2009.

[20] A. Noh, W. J. Lee, and J. Y. Ye, "Comparison of the mechanisms of the zigbee's indoor localization algorithm," in ACIS Int. Conf. on Software Engineering, Artificial Intelligence, Networking, and Parallel/Distributed Computing, Thailand, 2008.

[21] L. Marques, N. Almeida, and A. T. de Almeida, "Olfactory sensory system for odour-plume tracking and localization," in IEEE Int. Conf. on Sensors, Canada, 2003.

[22] A. Marjovi, J. Nunes, L. Marques, and A. T. de Almeida, "Multi-robot exploration and fire searching," in IEEE/RSJ Int. Conf. on Intelligent Robots and Systems, USA, 2009.

[23] C. J. Rodas, J. Escudero and D. I. Iglesia, "Bayesian filtering for a bluetooth positioning system," in IEEE Int. Symp. on Wireless Communication Systems, Iceland, 2008.

[24] J. Pascoal, P. Sousa, and L. Marques, "Khenose - a smart transducer for gas sensing," in Int. Conf. on Climbing and Walking Robots and the Support Technologies for Mobile Machines, Coimbra, Portugal, 2008.

[25] T. Lochmatter, X. Raemy, L. Matthey, S. Indra, and A. Martinoli, "A comparison of casting and spiraling algorithms for odor source localization in laminar flow," in IEEE Int. Conf. on Robotics and Automation, California, USA, 2008.

[26] B. Gerkey, R. T. Vaughan, and A. Howard, "The player/stage project: Tools for multi-robot and distributed sensor systems," in 11th Int. Conf. on Advanced Robotics, Portugal, 2003. 